\title{
Una secuencia de gestos funerarios manteños en la provincia de Manabi: primeros resultados de la tola J7, sitio de Japoto (Ecuador)
}

Une séquence de gestes funéraires manteños dans la province de Manabi: premiers résultats des recherches sur la tola J7, site de Japoto (Équateur) A sequence of funerary patterns from the manteña culture in the province of Manabi: preliminary results from tola J7, site of Japoto (Ecuador)

Tania Delabarde

\section{(2) OpenEdition}

\section{Journals}

Edición electrónica

URL: http://journals.openedition.org/bifea/3829

DOI: 10.4000/bifea.3829

ISSN: 2076-5827

Editor

Institut Français d'Études Andines

Edición impresa

Fecha de publicación: 1 diciembre 2006

Paginación: 313-320

ISSN: 0303-7495

Referencia electrónica

Tania Delabarde, « Una secuencia de gestos funerarios manteños en la provincia de Manabi: primeros resultados de la tola J7, sitio de Japoto (Ecuador) », Bulletin de l'Institut français d'études andines [En línea], 35 (3) | 2006, Publicado el 01 junio 2007, consultado el 01 diciembre 2020. URL : http:// journals.openedition.org/bifea/3829; DOI : https://doi.org/10.4000/bifea.3829

\section{(c) (i) $\odot$}

Les contenus du Bulletin de l'Institut français d'études andines sont mis à disposition selon les termes de la licence Creative Commons Attribution - Pas d'Utilisation Commerciale - Pas de Modification 4.0 International. 


\title{
Una secuencia de gestos funerarios manteños en la provincia de Manabi: primeros resultados de la tola $J 7$, sitio de Japoto (Ecuador)
}

\author{
Tania Delabarde*
}

\begin{abstract}
Resumen
Tras dos temporadas de excavación en la tola J7 se descubrió un asentamiento funerario de la cultura manteña. Los restos humanos fueron encontrados en depósitos primarios (tumbas) o secundarios (paquetes). La organización de los vestigios óseos y materiales reflejan una secuencia compleja de prácticas funerarias que van desde entierros de cuerpos completos hasta huesos desarticulados en paquetes. En Ecuador, dos sitios fechados en el periodo precerámico (Las Vegas) y Formativo (Real Alto) presentan depósitos parecidos. Sin embargo, el asentamiento funerario encontrado en la tola J7 es inédito hasta hoy en día para la cultura manteña.
\end{abstract}

Palabras clave: asentamiento funerario, tola, periodo precerámico

\section{Une séquence de gestes funéraires manteños dans la province de Manabi: premiers résultats des recherches sur la tola J7, site de Japoto (Équateur)}

\section{Résumé}

Les deux campagnes de fouille sur la tola $\mathrm{J} 7$ ont permis de mettre à jour un ensemble funéraire appartenant à la culture manteña. Les restes humains se trouvaient dans des dépôts primaires (tombe) ou secondaires (paquets). L'organisation des vestiges osseux et matériels reflète une séquence complexe de gestes funéraires autour du corps complet et jusqu'au paquet d'os. En Équateur, deux sites publiés présentent des dépôts similaires: le premier date de la période précéramique (Las Vegas) et le second de la période formative (Real Alto). Néanmoins, l'ensemble funéraire découvert à Japoto est à ce jour inédit pour la culture manteña.

Mots clés: ensemble funéraire, tola, période précéramique 


\title{
A sequence of funerary patterns from the manteña culture in the province of Manabi: preliminary results from tola $\mathrm{J} 7$, site of Japoto (Ecuador)
}

\begin{abstract}
After two field seasons in tola $\mathrm{J7}$, a funerary area attributed to the manteña culture was discovered. Human remains were found in primary (burials) and secondary deposits (packs). The distribution of bones and artifacts reflect a sequence of complex funerary practices that spans from the inhumation of complete corpses to packs of bones. In Ecuador only two other sites show similar features; one from the Preceramic period (Las Vegas) and one from the Formative period (Real Alto). However, the funerary place discovered in Japoto is to date, unknown to the manteña culture.
\end{abstract}

Key words: funerary place, tola, Formative period

\section{INTRODUCCIÓN}

A pesar de su riqueza arqueológica (Estrada, 1957; 1962), la costa central de Manabi no ha conocido grandes proyectos de investigación en los últimos 40 años como los que se han desarrollado en las provincias vecinas de Esmeraldas (Bouchard \& Usselmann, 2003; Guinea, 1995) o Guayas (Guillaume-Gentil, 1999; Stothert, 1988; Ubelaker, 1981). El sitio de Japoto, el único complejo de tolas ${ }^{1}$ conservado en esta costa, ofrece una nueva perspectiva de las ocupaciones prehispánicas de la cultura manteña en este litoral. Los manteños ocuparon la región de Manabi, desde el sur de Manta hasta Bahía de Caráquez a partir de 700 d.C. hasta la conquista española. La presencia de estos grupos al norte y también al sur de Ecuador (provincia de Guayas) originó la hipótesis de un gran complejo regional compuesto por los manteños del Norte y los manteños del Sur. Los asentamientos funerarios y el material excavado sirvieron como base para esa hipótesis. Sin embargo, las estructuras funerarias encontradas en el sitio de Japoto, y más específicamente en la tola J7, entregan datos inéditos sobre las prácticas de estas poblaciones del Periodo de Integración.

\section{PRESENTACIÓN Y METODOLOGÍA}

La tola J7 se ubica en el sitio de Japoto entre las tolas J3-J4 y la tola J5, a lo largo del lindero este de una finca de 25 hectáreas $^{2}$. Su forma es ovalada y presenta una cumbre convexa. Su eje longitudinal mide $36,48 \mathrm{~m}$, el eje norte-sur mide $30,94 \mathrm{~m}$ y tiene $3,60 \mathrm{~m}$ de altura en su cumbre. La estratigrafía es muy diferente de las tolas vecinas porque yace sobre un nivel de roca madre. Se puede hablar de un montículo semi-artificial considerando el hecho que los hombres aprovecharon una elevación natural para instalarse. Tres niveles fueron definidos: el primer nivel corresponde a la tierra vegetal donde se encontraron tiestos de cerámica; el segundo es una tierra de color amarilla-gris donde se hallaron la mayoría de los vestigios humanos y materiales. El último nivel es amarillo con guijarros provenientes de la descomposición del nivel estéril geológico. Aquí se encontraron los depósitos más profundos. Generalmente, los huesos humanos aparecen en el nivel 2 y la base del depósito yace en el nivel 3 geológico. Las características geomorfológicas de la tola $\mathrm{J} 7$ constituyen una particularidad que trataremos de relacionar con su funcionamiento.

1 Las tolas designan montículos artificiales.

2 La propiedad pertenece a la señora Guadalupe Castro. 
Durante la primera prospección en la tola $\mathrm{J} 7$, se observaron varias concentraciones de restos humanos muy fragmentados en la superficie de la vertiente norte. Una excavación en área permitió tener una idea del tipo de ocupación de esa parte de la tola. Todos los vestigios encontrados son depósitos funerarios. Las huellas de poste y las concentraciones de cerámica y/o de ceniza parecen asociadas al contexto funerario. La mayoría de los depósitos funerarios son secundarios pero se encontraron algunos entierros primarios. Hemos utilizado el registro y levantamiento de huesos por niveles para poder demostrar la complejidad y la organización de los depósitos secundarios que contienen hasta 7 individuos. Además, el registro y el dibujo de cada hueso en el campo, su cara de aparición y su orientación fueron necesarios considerando la mala preservación de los mismos. Los depósitos funerarios se encuentran desde la superficie o muy cerca de la misma (entre 5 y $10 \mathrm{~cm}$ ). Los vestigios han sufrido las actividades humanas, animales y por supuesto las intemperies. El análisis de los restos humanos de la tola J7 y del sitio de Japoto en general nos permitió establecer una aproximación biológica a la población. Sin embargo, en la mayoría de los casos, la mala preservación de los restos óseos no permitió la individualización de los difuntos. Se calculó el Número Mínimo de Individuo (NMI) en base al hueso más frecuente y con un sistema de asociaciones y exclusiones. Para determinar la edad de los adultos, se utilizaron las técnicas de la metamorfosis de la sínfisis púbica (Brooks \& Suchey, 1990) y de la cuarta costilla (Iscan et al., 1985). Para los niños se observó la erupción dental (Ubelaker, 1999) y el desarrollo óseo (Scheuer \& Black, 2000).

\section{INVESTIGACIONES Y RESULTADOS PRELIMINARES}

La tola 7 fue excavada durante dos temporadas $(2004,2006)$ de cinco semanas de trabajo en el campo. Mediante la recolección superficial de fragmentos de huesos humanos, tres zonas (Z1, Z2 y Z3) fueron inicialmente definidas (fig. 1). En 2004, se excavó un área de $21 \mathrm{~m}^{2}$ para verificar la presencia de tumbas en esta ubicación. Cerca de la zona 1, se encontraron algunos huesos humanos y una amplificación al norte de la misma permitió descubrir un depósito secundario (Z0) y un entierro primario (ZOB).

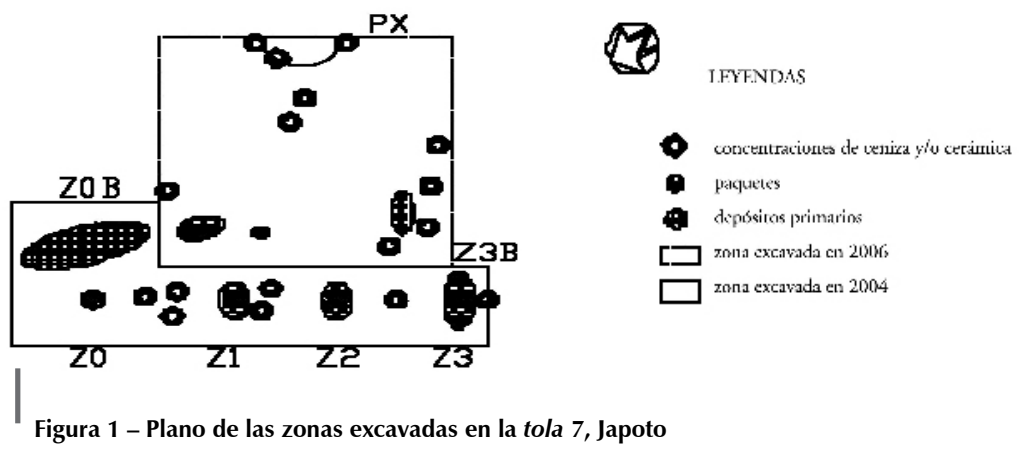

Algunos huesos sin conexión anatómica, organizados en un paquete fueron hallados en el sector Z0 (una mandíbula, un tercer metatarsiano izquierdo, un cuarto metatarsiano izquierdo, una vértebra cervical ${ }^{3}$, un fragmento de vértebra torácica, fragmentos de diáfisis de hueso largo). La zona $\mathrm{OB}$ reveló un entierro primario de un individuo adulto, que carece de cráneo. Sin embargo su presencia inicial es conocida por la presencia de dientes del maxilar y de la mandíbula ${ }^{4}$

3 La vértebra cervical es la cuarta o quinta.

4 Los dientes encontrados en la tumba no pertenecen a la mandíbula del paquete secundario. 
Considerando que los restos post cráneos 5 del difunto están completos, se puede decir que los huesos encontrados en el paquete Z0 pertenecen a otro individuo. El difunto, un individuo adulto de sexo masculino, descansaba en posición decúbito dorsal orientado en un eje sur-norte (pies al norte) en una fosa de 2,1 m largo x $60 \mathrm{~cm}$ de ancho con los miembros superiores e inferiores extendidos en el eje del cuerpo. Ninguna ofrenda fue rescatada a pesar de que algunas cuentas y un anillo de concha fueron encontrados en el relleno de la fosa.

En la zona 1 se encontró un entierro secundario con los restos de tres individuos adultos, dos con morfología robusta (¿masculinos?) y uno menos robusta (¿femenino?). Los huesos están organizados en un paquete de $60 \mathrm{~cm}$ de largo por $30 \mathrm{~cm}$ de ancho. Los primeros niveles presentan un cráneo6 y cuatro huesos largos y luego los huesos del tórax y de las extremidades. En el último nivel se registraron dos antebrazos articulados con las manos que podrían pertenecer a uno de los individuos robustos. El individuo 1 se encontró casi completo mientras los otros dos individuos están solo representados por algunos huesos. El NMI7 fue calculado en base a tres radios derechos.

La zona 2 reveló la presencia de tres cráneos pertenecientes a dos adultos y un niño en un paquete de $75 \mathrm{~cm}$ de largo por $60 \mathrm{~cm}$ de ancho. De nuevo los cráneos y los huesos largos 8 aparecieron en el primer nivel mientras los restos de la columna y las extremidades se hallaron en los últimos niveles (fig. 2). Los dos cráneos de adulto aparecieron uno mirando al este mientras el segundo mirando al oeste. El cráneo del niño se encontró muy quebrado y su posición no pudo ser determinada. Una aguja de cobre y varias cuentas de concha estaban en el primer nivel con los cráneos.

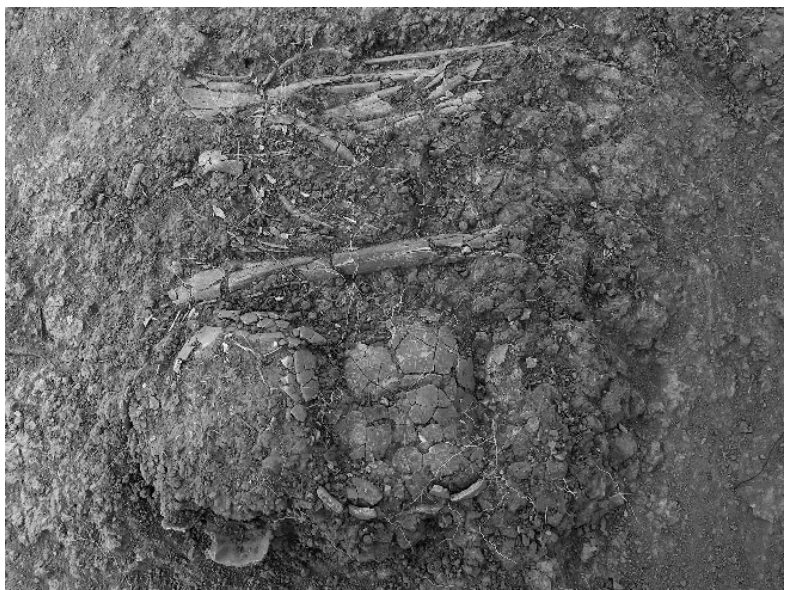

Figura 2 - Paquete secundario de la zona 2 nivel 1, tola 7, Japoto

Se encontró otro depósito secundario en la zona 3 con un número mínimo de cinco adultos y dos niños ${ }^{9}$. El paquete mide $60 \mathrm{~cm}$ de largo por $30 \mathrm{~cm}$ de ancho. Dos cráneos (un adulto y un niño) y una mandíbula 10 robusta aparecieron en el primer nivel. Los huesos largos aparecen alineados según un eje esteoeste. La particularidad de este depósito es la presencia de estos huesos desde el primer nivel hasta el último. Los huesos de la columna y de las extremidades se encontraron en los últimos niveles. Un tortero fue registrado en el nivel superficial.

En 2006, aprovechamos el pozo de huaquero de la parte suroeste para determinar la estratigrafía general de la tola. Encontramos los mismos niveles que en la zona excavada en 2004 y se confirmó la presencia de un montículo natural sobre la roca madre. Se abrió una cuadrícula de $16 \mathrm{~m}^{2}$ al este de la zona excavada en 2004, en la parte alta de la tola, con el objetivo de obtener más datos sobre su funcionamiento y verificar la hipótesis de un asentamiento funerario, considerando la concentración de restos humanos hallados en esta zona restringida.

5 Este término anatómico designa todos los huesos del cuerpo a excepción del cráneo y la mandíbula.

6 El término cráneo es diferente del término esqueleto cefálico (cráneo y mandíbula).

7 Número mínimo de individuo.

8 Dos fémures y un húmero aparecieron empilados según el mismo eje.

9 El Número Mínimo de Individuo se calculó en base a la presencia de 5 fémures derechos y otros huesos de dos niños de edad diferente.

10 No se pudo establecer si la mandíbula pertenecía o no al cráneo encontrado en el mismo nivel por la mala conservación de este último. 
Esta área nos permitió descubrir otro conjunto de restos humanos (un entierro primario y dos depósitos secundarios) asociado a unos pozos de combustión y concentraciones de cerámica. Como en 2004, la cerámica no se encontró dispersa y abundante como en las tolas vecinas, pero en concentraciones de tiestos de una o varias vasijas asociadas a los pozos de ceniza dentro de los cuales se hallaban (o no) algunos tiestos. Sin embargo no se encontró ningún resto óseo de fauna ni tampoco restos humanos. Estos pozos encontrados en 2004 y 2006 podrían ser asociados al asentamiento funerario más que a instalaciones domésticas.

Las concentraciones de cerámica se encontraron en el segundo nivel, así como una figura de rana en cerámica que apareció en la parte suroeste de la cuadrícula. Después del levantamiento de este nivel y al seguir excavando debajo de la rana, apareció una estatua11 de mujer embarazada asociada a los restos de un niño en conexión anatómica parcial. El pequeño difunto (E1) descansaba en posición decúbito dorsal con los miembros inferiores extendidos a lo largo del cuerpo. No se pudo observar evidencias de una fosa, pero en base a la desarticulación de los huesos se infiere que el cuerpo no fue enterrado sino depositado con la estatua de mujer embarazada en el nivel 3 (fig. 3).

La mayoría de los restos humanos descubiertos en la tola 7 se encontraron en este mismo nivel así como los pozos de ceniza. El análisis de los restos óseos no permitió determinar la causa de muerte pero la edad pudo ser estimada entre 18 y 24 meses en base a la medida de los huesos largos y de la erupción dental. Este depósito constituye la primera tumba con ajuar funerario encontrada en el sitio de San Jacinto. Nuestra hipótesis sobre este conjunto sería que puede tratarse de un conjunto funerario tridimensional. La estatua de mujer embarazada y la figura de rana pueden representar la relación entre los dos protagonistas de este trágico evento, la mujer embarazada siendo la madre y la rana el juguete del niño o un símbolo de fertilidad.

En la parte noreste de la cuadrícula, un depósito secundario (E2) apareció en el nivel 3. Se trata de un paquete organizado con los huesos del esqueleto axial12 y de las extremidades (manos y pies) en los primeros niveles (fig. 4), y luego, los huesos largos empilados en el mismo eje (norte-sur) (fig. 5). Aparentemente no había conexiones anatómicas, pero al levantar y dibujar los huesos se notó algunas, y en los últimos niveles se encontró un antebrazo derecho (radio y cúbito) en conexión anatómica estricta con algunos huesos de la mano. Los huesos largos se encontraron divididos en lados, con el fémur, tibia, húmero,

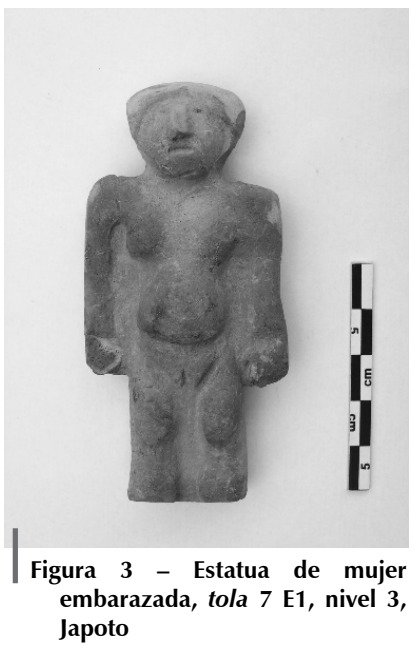
radio y ulna izquierdo de un lado y los derechos del otro lado (fig. 5-6). La ubicación de los fémures, tibia y fíbula era similar a la posición de una pierna doblada, como si los huesos de los miembros inferiores hubieran sido doblados a partir de los restos de un difunto completo y en conexión anatómica. Sin embargo, la patella izquierda apareció a $36 \mathrm{~cm}$ de la epífisis proximal del fémur izquierdo, pero se trata del único hueso encontrado aislado. La organización y la ubicación de los restos óseos del paquete E2 reflejan la posible secuencia de unos depósitos funerarios y demuestra la complejidad de las prácticas de los manteños.

Mediante el análisis del paquete secundario E2 se concluyó que los restos óseos pertenecen a un solo individuo adulto de sexo masculino. El esqueleto es casi completo a excepción de la cabeza. No se pudo calcular la estatura, considerando el estado de preservación de los huesos. La edad se estimo entre 35 y 45 años. Las vértebras torácicas y lumbares presentan evidencias de osteofitis que se pueden relacionar con una actividad especial (transporte de carga) más que con la edad. Otro paquete llevando algunos huesos (E3) fue excavado a $53 \mathrm{~cm}$ al sureste del depósito E2. Dentro de este depósito13, se encontró una patella derecha pero su morfología era

11 La estatua está hecha en cerámica.

12 Este término designa los huesos de la columna, tórax y cintura pélvica.

13 El paquete E3 lleva los huesos siguientes: una patella derecha, fragmentos de manubrio, la primera, la segunda y la tercera cervical, un fragmento de diáfisis de costilla derecha, un cuneiforme medial derecho, una premolar con una caries en la cara mesial. 


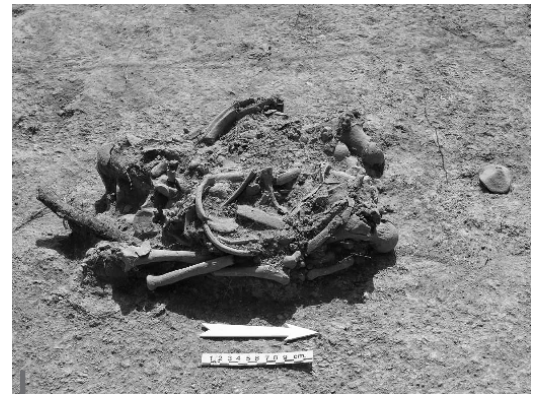

Figura 4 - Paquete secundario E2, nivel 2, tola 7, Japoto

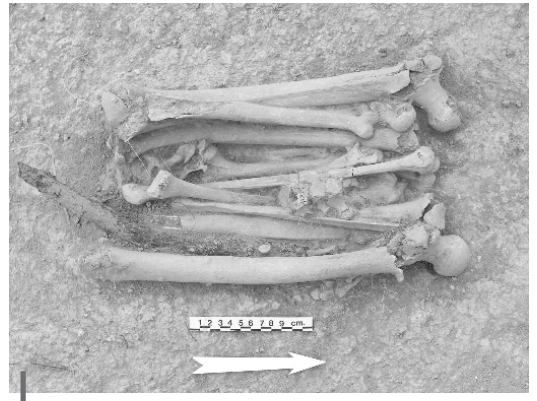

Figuras 5 - Paquete secundario E2, nivel 6, tola 7, Japoto

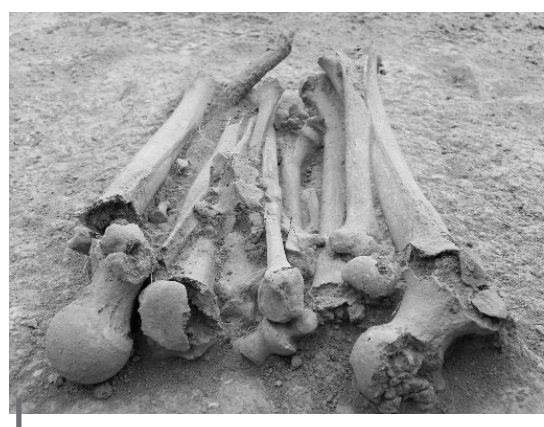

Figura 6 - Paquete secundario E2, nivel 6, tola 7, Japoto

muy distinta a la del paquete E2. Además, la presencia de las tres primeras vértebras cervicales ${ }^{14}$ en conexión anatómica nos permitió concluir que este paquete representa otro individuo de sexo indeterminado.

Los tres depósitos funerarios hallados en 2006 presentan un Número Mínimo de Individuo de 2 adultos y un niño. Los depósitos contienen un solo individuo. Los difuntos están completos (E1) o representados por algunos huesos (E3). Sin embargo, se encontró por primera vez en el sitio de Japoto ofrendas funerarias (figuras de cerámica) directamente asociadas con el difunto. Estos depósitos encontrados durante las dos temporadas comparten muchas características (tipo de depósito, organización, repartición de los restos óseos) y serían parte de un mismo asentamiento funerario, cuyos límites y extensión faltan por definir. La zona excavada en 2006 fue cerrada y protegida con el objetivo de terminar su excavación en las próximas temporadas.

\section{DISCUSIÓN Y PERSPECTIVAS DE TRABAJO EN EL FUTURO}

La excavación de la tola $\mathrm{J} 7$ permitió descubrir en una zona restringida de $37 \mathrm{~m}^{2}$ una concentración importante de restos humanos ( $\mathrm{NMI}=18$ ) en depósitos primarios (2) y secundarios (6) asociados a unos pozos y concentraciones de cerámica (cuadro 1). Las características de estos vestigios y la diferencia con los descubrimientos en las tolas vecinas, tanto en la estratigrafía como en el material permiten hablar de un asentamiento funerario.

\footnotetext{
14 Se encontró también la primera y la segunda cervical en conexión anatómica dentro del paquete E2.
} 
Una secuencia de gestos funerarios manteños en la provincia de Manabi

Cuadro 1 - Cuadro con los depósitos funerarios y los individuos encontrados en la tola 7, Japoto

\begin{tabular}{|l|l|l|l|l|}
\hline Temporada & Depósitos funerarios & Individuos & \multicolumn{1}{c|}{ Sexo } & \multicolumn{1}{c|}{ Edad } \\
\hline 2004 & Secundario (Z0) & $\mathrm{NMI}=1$ & Indeterminado & Adulto \\
\hline & Primario (ZOB) & 1 & Masculino & $30-50$ años \\
\hline & Secundario (Z1) & $\mathrm{NMI}=3$ & Indeterminados & 3 Adultos \\
\hline & Secundario (Z2) & $\mathrm{NMI}=3$ & Indeterminados & $\begin{array}{l}2 \text { Adultos } \\
1 \text { Niño }\end{array}$ \\
\hline & Secundario (Z3) & $\mathrm{NMI}=7$ & Indeterminado & $\begin{array}{l}5 \text { Adultos } \\
2 \text { Niños }\end{array}$ \\
\hline & Primario (E1) & 1 & Indeterminado & 18 -24 meses \\
\hline & Secundario (E2) & $\mathrm{NMI}=1$ & Masculino & $30-50$ años \\
\hline & Secundario (E3) & $\mathrm{NMI}=1$ & Indeterminado & Adulto \\
\hline TOTAL & $\begin{array}{l}\text { 2 Primarios } \\
\text { 6 Secundarios }\end{array}$ & $\mathrm{NMI}=18$ & $\begin{array}{l}\text { 2 Masculinos } \\
16 \text { Indeterminados }\end{array}$ & \\
& 8 Depósitos & & & \\
\hline
\end{tabular}

La variabilidad de los depósitos (primarios, secundarios), la organización de los restos óseos (tumba, paquete, orientación) y la representación de cada difunto (completo, incompleto, representado por algunos huesos) reflejan una secuencia de gestos funerarios en una zona específica donde se depositaban los cadáveres completos, los cuales podrían ser luego organizados en paquetes secundarios. El material cerámico asociado a los restos humanos es característico de la cultura manteña. Otros entierros primarios y paquetes secundarios se hallaron en las tolas $\mathrm{J} 5$ y $\mathrm{J} 7$ pero estos depósitos estaban menos numerosos y asociados con asentamientos domésticos. La particularidad de la tola 7 es la cantidad y la variabilidad de los depósitos y hasta hoy en día la presencia exclusiva de estructuras funerarias.

Una síntesis de los sitios funerarios costeños del Ecuador precolombino menciona dos sitios documentados en la costa sur con este tipo de práctica funeraria (Delabarde, 1997). Se trata del cementerio precerámico OGSE 80 Las Vegas ubicado en la península de Santa Elena donde hallaron paquetes secundarios asociados a entierros primarios y cráneos aislados asociados a huesos largos (Stothert, 1988). Los reportes de investigaciones del sitio de Real Alto mencionan un solo depósito parecido, pero los datos no son detallados (Marcos, 1988). Sin embargo, estos dos sitios son muy distintos al nivel cronológico como cultural del sitio de Japoto y hasta hoy en día, no existen datos comparables en la literatura arqueológica sobre los manteños.

En Japoto, solo una amplificación de la zona excavada permitirá determinar si la tola J7 ha sido específicamente funeraria. Muestras del nivel de roca madre fueron colectadas y esperemos establecer si existen unas propiedades geomorfológicas relacionadas con la preservación de los cuerpos o al revés para acelerar la descomposición de los mismos. Planificamos mandar una serie de muestras para comparar los ADN de los difuntos de la tola J7 y establecer si existe una filiación entre los mismos y también con los individuos encontrados en las diferentes tolas de Japoto. Sería además importante establecer si el cráneo ausente del entierro primario Z0B de la tola 77 se encuentra en unos de los paquetes secundarios vecinos. Para obtener más datos sobre la dieta de estas poblaciones, procederemos a unos análisis de elementos traces y comparar los resultados con los de nuestra colega Mercedes Guinea obtenidos a partir de las empanadillas encontradas en las diferentes tolas de Japoto. Al seguir el estudio de este sitio y con los resultados 
de los diferentes análisis, esperemos obtener más datos biológicos, culturales y cronológicos sobre las poblaciones manteñas, cuyos comportamientos frente a la muerte fueron tan complejos.

\section{Referencias citadas}

BOUCHARD, J.-F. \& USSELMANN, P., 2003 - La région Tumaco-La Tolita : cultures préhispaniques et modernes, 154 p.; Paris: CNRS éditions.

BROOKS, S. T. \& SUCHEY, J. M., 1990 - Skeletal age determination based on the os pubis: A comparison of the Ascáadi-Nemeskeri and Suchey-Brooks methods. Human Evolution, 5: 227-238; Academic Press.

DELABARDE, T., 1997 - Les sépultures de la côte centre sud de l’Équateur : Unité et diversité des gestes funéraires. Mémoire de Maîtrise de I’Université de Paris I, la Sorbonne. Manuscrito.

ESTRADA, E., 1957 - Prehistoria de Manabí, 176 p.; Guayaquil: Archivo Histórico del Guayas.

ESTRADA, E., 1962 - Arqueología de Manabí central, 205 p.; Guayaquil: Museo Victor Emilio Estrada.

GUILLAUME-GENTIL, N., 1999 - Les tolas du nord du bassin du Guayas : éléments de chronologie et modèles d'occupation. In: Actes des Journées d'études d'archéologie précolombienne: Amérique du Sud, des chasseurs cueilleurs à l'Empire Inca, Genève 10 et 11 octobre 1997: 107-128; Oxford: BAR International Series 756.

GUINEA, M., 1995 - Diferentes mecanismos de articulación Hombre-entorno en la costa norte del Ecuador. La desembocadura de Esmeradas del principio de nuestra era hasta el año 1527. In: Primer Encuentro de Investigadores de la costa ecuatoriana en Europa: 47-66; Quito: Abya-Yala.

ISCAN, M. Y., LOTH, S. R. \& WRIGHT, R. K., 1985 - Age estimation from the rib by phase analysis: White Males. Journal of Forensics Sciences, 30: 853-863; Colorado: American Academy of Forensic Sciences.

MARCOS, J. G., 1988 - Real Alto: la historia de un centro ceremonial Valdivia (primera parte); Quito: Escuela Politécnica del Litoral, Centro de Estudios Arqueológicos y Antropológicos, Corporación Nacional. Biblioteca Ecuatoriana de Arqueología 4.

SCHEUER, L. \& BLACK, S., 2000 - Developmental Juvenile Osteology, 587 p.; USA: Academic Press Inc.

STOTHERT, K. E., 1988 - La prehistoria temprana de la península de Santa Elena, Ecuador: Cultura Las Vegas, 271 p.; Guayaquil: Banco Central del Ecuador.

UBELAKER, D. H., 1981 - The Ayalán Cemetery: A Late Integration Period Burial Site on the south coast of Ecuador, 175 p.; Washington: Smithsonian Contributions to Anthropology 29.

UBELAKER, D. H., 1999 - Human skeletal remains, 172 p.; Washington: Taraxacum Press. 